\title{
Analysis of the intra- and intertumoral heterogeneity of hypoxia in pancreatic cancer patients receiving the nitroimidazole tracer pimonidazole
}

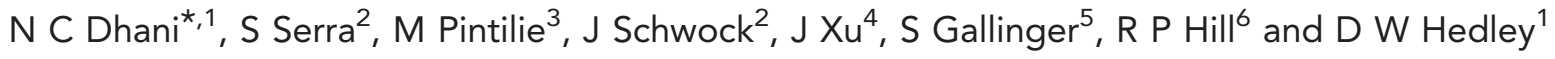

${ }^{1}$ Division of Medical Oncology and Hematology, University Health Network, Princess Margaret Cancer Centre/Ontario Cancer Institute, 610 University Avenue, Toronto, ON, Canada M5G 2M9; ${ }^{2}$ Department of Laboratory Medicine and Pathobiology, University Health Network, Princess Margaret Cancer Centre/Ontario Cancer Institute, 610 University Avenue, Toronto, ON, Canada M5G 2M9; ${ }^{3}$ Department of Biostatistics, University Health Network, Princess Margaret Cancer Centre/Ontario Cancer Institute, 610 University Avenue, Toronto, ON, M5G 2M9; ${ }^{4}$ Applied Molecular Profiling Laboratory, University Health Network, Princess Margaret Cancer Centre/Ontario Cancer Institute, 610 University Avenue, Toronto, ON, Canada M5G 2M9; ${ }^{5}$ Division of Hepato-biliary Pancreatic Surgical Oncology, University Health Network and Mount Sinai Hospital, University Health Network, Princess Margaret Cancer Centre/ Ontario Cancer Institute, 610 University Avenue, Toronto, ON, Canada M5G 2M9 and ${ }^{6}$ Radiation Medicine Program, University Health Network, Princess Margaret Cancer Centre/Ontario Cancer Institute, 610 University Avenue, Toronto, ON, Canada M5G 2M9
\end{abstract}

Background: Hypoxia is thought to be an adverse feature of pancreatic cancer, but direct measurement in patients is technically challenging. To address this, we characterised the intra/interpatient heterogeneity of hypoxia in surgical specimens from patients who received the 2-nitroimidazole tracer pimonidazole pre-operatively.

Methods: Pimondazole was given intravenously 16-20 h before pancreatectomy, and the extent and intratumoral heterogeneity of hypoxia determined by image analysis applied to multiple tissue blocks stained by immunohistochemistry. Intra/interpatient heterogeneity was estimated by variance component analysis.

Results: Pimonidazole staining was analysed in 10 tumours. The extent of labelling varied amongst patients (0-26\%), with a broader range of hypoxia in the epithelial (1-39\%) compared with the stromal (1-13\%) compartments. Variance component analysis demonstrated greater inter- than intrapatient variability of hypoxia, and that multiple (4-5) tumour sections are required to provide a consistent evaluation of its extent in individual tumours.

Conclusions: There is significant intra- and intertumoral heterogeneity of hypoxia in pancreatic cancers, and these do not appear to be generally more hypoxic than other cancer types. This study establishes the feasibility to assess hypoxia in pancreatic cancer patients using pimonidazole, but questions the reliability of measurements made using a single tissue section.

Pancreatic ductal adenocarcinoma has the poorest treatment outcome of the common cancers, and current projections estimate that it will become the second leading cause of cancer death in western countries after lung cancer by 2030 (Rahib et al, 2014).
Early whole-genome and targeted sequencing studies have partially revealed the extent of genetic complexity inherent in this disease, suggesting that highly specific targeting of molecular aberrations unique to epithelial malignant cells is unlikely to result in sustained 
benefit for large groups of patients (Jones et al, 2008; Alexandrov et al, 2013). Contributing additional complexity is the tumour stroma-a heterogenous collection of fibroblasts, immunomodulatory and vascular elements embedded in a biochemically diverse extracellular matrix (ECM) believed to be of particular relevance to pancreatic ductal adenocarcinoma (PDAC) tumorigenesis (Neesse et al, 2011).

The dense desmoplastic stroma that characterises PDAC has been proposed as a determinant of biological aggression, promoting invasion and metastasis as well as resistance to standard therapies (Olive et al, 2009; Provenzano et al, 2012). Several mechanisms have been suggested, including compromised tumour perfusion that limits drug penetration; the promotion of an acidotic, hypoxic microenvironment; and the activation of tumour-promoting signalling by ECM components such as collagen-1, the matrix metalloproteinases and TGF- $\beta$ (Armstrong et al, 2004; He et al, 2007; Olive et al, 2009; Neesse et al, 2011).

The development of microregional hypoxia in solid tumours is well described with higher levels of hypoxia being associated with poor patient outcome due to inferior treatment response and more aggressive tumour behaviour (Fyles et al, 2002; Brown and Wilson, 2004; Nordsmark et al, 2006; Rischin et al, 2006; Vaupel, 2008; Overgaard, 2011). PDAC has been historically considered unusually hypoxic, based on poor perfusion by radiological contrast agents ((Fusaroli et al, 2010; Matsubara et al, 2011) and Eppendorf electrode probe $\mathrm{pO}_{2}$ measurements made intraoperatively in a series of seven patients with resectable pancreatic cancers (Koong et al, 2000). Still, although preclinical data support tumoral hypoxia as a relevant factor in the aggressive biology of PDAC (Buchler et al, 2004; Spivak-Kroizman et al, 2013), this remains to be clinically validated.

Using orthotopically grown patient-derived xenograft models of PDAC, we observed a wide range in hypoxia, and a striking correlation between higher levels of hypoxia and increased cellular proliferation, tumour growth rate and greater metastatic potential (Chang et al, 2011). These results support the concept that hypoxia is associated with biological aggression of PDAC. To establish the clinical relevance of these findings, we initiated the PIMO-PANC trial (NCT01248637), which administers the 2-nitroimidazole hypoxia tracer pimonidazole to patients pre-operatively. Pimonidazole undergoes bioreductive metabolism under low oxygen conditions to form stable adducts that can be detected in tissue sections by immunohistochemistry (Raleigh et al, 1992; Varia et al, 1998) (Bussink et al, 2003). This ongoing study has a target accrual of 100 patients, and is powered to assess the prognostic importance of hypoxia in early stage pancreatic cancer. In the present paper, we describe an interim analysis of the intra/interpatient heterogeneity of hypoxia, and the importance of sampling error due to intratumoral heterogeneity when assessing tumour hypoxia using histological markers. Our results suggest that this is a larger problem than is often appreciated.

\section{MATERIALS AND METHODS}

Materials. Pimonidazole hydrochloride (Hypoxyprobe-1) was obtained from Natural Pharmacia International (NPI) Inc, Belmont, MA, USA and has IND (Investigational New Drug) status for use for the clinical evaluation of hypoxia. Immunohistochemical (IHC) detection of stable pimonidazole protein adducts was performed using (mouse) monoclonal $\mathrm{IgG}_{1}$, Hypoxyprobe MAb1 (Raleigh et al, 1992; Raleigh et al, 1999), biotin blocking kit (Vector Lab, Burlington, ON, Canada), streptavidin biotin detection (Signet Pathology System, Deham, MA, USA), Nova Red substrate (Vector Lab), Mayer's haematoxylin counterstaining and Permount mounting medium.
Patients. Approval was obtained from the University Health Network Research Ethics Board. The eligible patient cohort included those scheduled for surgery with a presumptive diagnosis of pancreatic adenocarcinoma. Male and female patients, over 18 years of age and of all ethnicities, were included. Written informed consent was obtained before patient registration.

Drug administration. Enroled patients received one dose of intravenous pimonidazole $\left(0.5 \mathrm{gm} \mathrm{m}^{-2}\right)$ over a minimum of $30 \mathrm{~min}$ on the day before surgery. Pimonidazole was administered on an out-patient basis under medical supervision with infusion being completed 16-20 h before surgery. Manufacturer's recommendations are for tumour sampling to take place at $16-24 \mathrm{~h}$ post pimonidazole administration ensuring minimal levels of circulating pimonidazole at the time of tumour harvesting. Pimonidazole labelling is therefore reflective of in vivo hypoxia status of tumour and not of hypoxia sustained during tumour harvesting.

Tissue processing. Resected tumours were processed for paraffin embedding and histological examination according to standard pathological practice, yielding multiple paraffin blocks from the primary tumour and regional lymph nodes. This material was reviewed (ND, SS), and blocks representative of primary tumour were selected. Serial sections were stained for haematoxylin and eosin (H\&E) and pimonidazole (monoclonal $\mathrm{IgG}_{1}$ antibody, Hypoxyprobe MAb1). The IHC protocol was followed as described previously and outlined below (Raleigh et al, 1998). Stained sections were digitised using an Aperio ScanScope XT (Aperio Technologies, Vista, CA, USA) whole slide scanner at $\times 20$ magnification (resolution $0.5 \mu \mathrm{M}$ per pixel).

Pimonidazole IHC. Unless specified, all steps were performed at ambient temperature with PBS washes between incubations. Paraffin sections were dried in a $60{ }^{\circ} \mathrm{C}$ oven overnight, dewaxed and endogenous peroxidase blocked by incubation in 3\% hydrogen peroxide for $10 \mathrm{~min}$. Following microwave antigen retrieval, endogenous biotin was blocked (Vector blocking kit). Slides were incubated overnight in pimonidazole mouse monoclonal antibody followed by biotinylated anti-mouse IgG, streptavidin biotin (30 min each) and Nova Red substrate ( $5 \mathrm{~min})$, then counterstained in Mayer's haematoxylin.

Histological tumour analysis. Image analysis included quantitation of (1) proportion of epithelial and stromal tumour compartments and (2) hypoxic fraction in whole tumour (i.e., in combined epithelial and stromal regions) as well as in individual compartments. 'Hypoxic tumour areas' were defined as regions demonstrating any positive immunostaining for pimonidazole. Images were managed on Spectrum, and analysis was completed using Genie, Aperio's pattern recognition software. This image analysis platform differentiates different histological features through computer-based learning algorithms by running repetitive training sequences using examples of different cellular morphologies to define 'classes.'

H\&E sections were first reviewed to identify appropriate tumour regions for analysis, excluding large areas of necrosis, atrophic pancreas and non-neoplastic tissues. A region of interest was manually selected for each pimonidazole IHC image based on H\&E. The Genie classes 'epithelium,' 'stroma' and 'other' were defined, with 'other' including necrosis, luminal spaces and any 'non-tumour' tissue to be excluded from analysis. On the basis of an observer-set threshold/training, a unique Genie classifier was developed for each patient's tumour in order to differentiate epithelial from stromal tumour compartments. Aperio's Positive Pixel v9 algorithm was applied to quantify hypoxic percentages in whole tumour with non-tumour tissue (including necrotic and luminal spaces) defined as 'other' being excluded and epithelial and stromal compartments separately. Microscopic scoring analysis was also conducted independently by one of the authors (JS), 
assigning each section a score (in 5\% increments) representing per cent hypoxia in the epithelial tumour compartment.

Statistical analysis. On the basis of our previous studies of the intratumoral heterogeneity of hypoxia, we estimated that an analysis of five sections per tumour in 10 patient tumours would provide a reasonable initial estimate of hypoxia levels in resected pancreatic cancers (Thrall et al, 1997; Iakovlev et al, 2007; Maseide et al, 2008; Pintilie et al, 2009). Hypoxia levels were measured in multiple sections of the same specimen (each from a different tissue block). Variance of hypoxia within and across tumours was calculated using variance component analysis. The intraclass correlation coefficient (ICC), or the per cent variation attributable to that between tumours, was also calculated from simulations. The ICC is a measure of reliability ranging between 0 and 1 . A value of 0.85 or higher is considered indicative of good reliability reflecting the fact that the variability between tumours explains most of the total variability. In contrast, low values for ICC reflect poor reliability suggesting that a large proportion of the total variance is due to the variance within a tumour. To estimate the incremental benefit of analysis of multiple sections per patient, we calculated the ICC for the average value of hypoxia for the scenarios when 1-6 sections per patient were to be analysed.

The relationship between hypoxia in epithelial and stromal compartments was assessed using the Spearman correlation coefficient.

\section{RESULTS}

Of the first 16 patients who received pimonidazole and proceeded to surgery, 5 patients were intraoperatively found to have advanced

\begin{tabular}{|c|c|c|c|}
\hline $\begin{array}{c}\text { Patient } \\
\text { ID }\end{array}$ & $\begin{array}{c}\text { Sections } \\
\text { anlaysed }\end{array}$ & $\begin{array}{c}\text { Mean epithelial \% } \\
\text { (range) }\end{array}$ & $\begin{array}{c}\text { Mean stromal \% } \\
\text { (range) }\end{array}$ \\
\hline 001 & 5 & $15(13-19)$ & $78(72-87)$ \\
\hline 002 & 6 & $26(16-41)$ & $65(53-81)$ \\
\hline 003 & 6 & $19(13-24)$ & $67(54-78)$ \\
\hline 004 & 3 & $26(21-36)$ & $66(59-74)$ \\
\hline 007 & 7 & $39(19-52)$ & $53(41-71)$ \\
\hline 008 & 6 & $50(36-64)$ & $38(25-56)$ \\
\hline 010 & 6 & $39(11-46)$ & $53(41-83)$ \\
\hline 012 & 7 & $26(8-35)$ & $60(51-86)$ \\
\hline 014 & 6 & $17(10-25)$ & $76(74-86)$ \\
\hline 017 & 5 & $44(31-60)$ & $42(33-51)$ \\
\hline
\end{tabular}

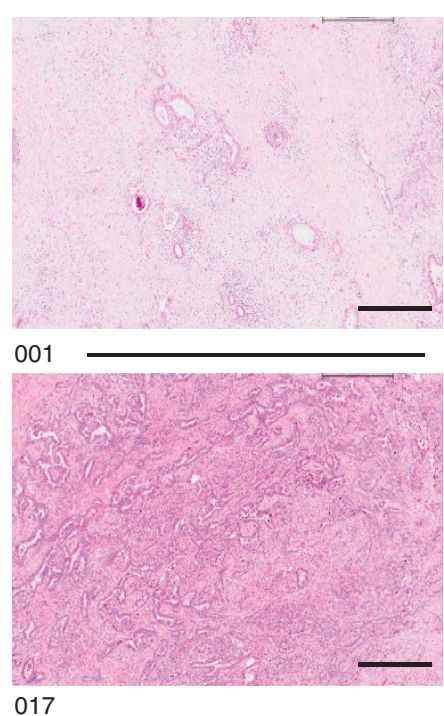

Figure 1. Heterogeneity of extent of stroma across patient tumours. The table outlines stromal content across 10 resected PDACs represented as mean value across all tumour sections analysed. Included histology are representative images of tumours with high (001: 78\%) and moderate (017: 42\%) stromal content; scale bar, $500 \mu \mathrm{m}$.

Normal pancreas (002)
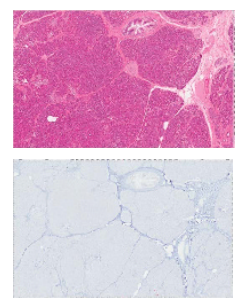

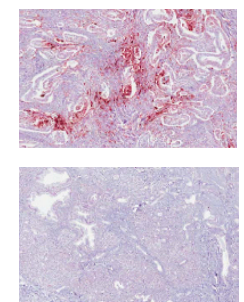


Figure 2. Representative histology (H\&E (columns 2 and 4) and pimonidazole immunohistochemistry (IHC) (columns 3 and 5$)$ ) for 10 resected tumours included in heterogeneity analysis. (H\&E for individual patients are labelled with patient study ID (001 to 017) with corresponding pimonidazole IHC in column to right). Note absence of pimonidazole staining in adjacent normal pancreatic tissue (column 1). 
disease precluding definitive resection. In these patients with locally advanced or metastatic disease, biopsies taken to confirm/establish histological diagnosis were evaluated qualitatively for pimonidazole staining. Regions of hypoxia, as indicated by positive pimonidazole staining, were observed in the liver and lymph node metastases (Supplementary Fig 1). The group of 11 resected tumours contained 1 common bile duct cancer, leaving 10 PDACs that are the subject of this study. Relevant clinicopathologic characteristics of these patients/tumours are outlined in Supplementary Table 1. The majority (8 out of 10 ) had a primary tumour located in the head of the pancreas, and (9 out of 10) had lymph node involvement. Four out of the 10 patients completed neoadjuvant treatment before surgery3 received 2 months of gemcitabine and erlotinib on a

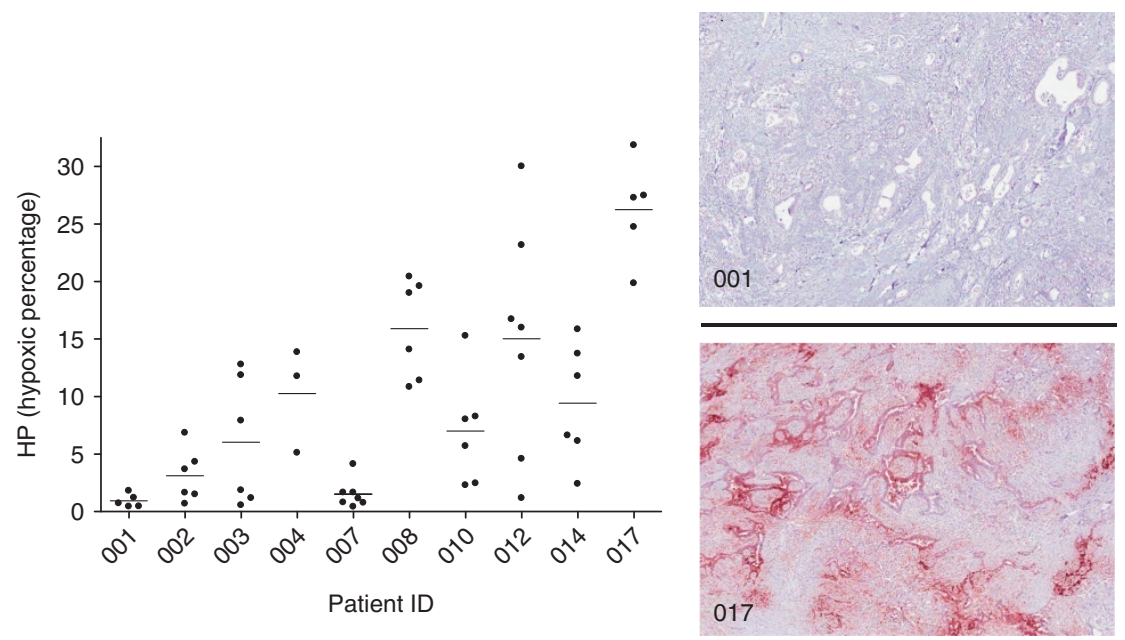

Figure 3. Hypoxic percentage (HP) in whole tumour (i.e., including both epithelial and stromal tumour components) defined as positive pimonidazole staining per whole-tumour section analysed. Each dot represents the HP measured in a single section and the bar the mean value from analysis of all sections.

\begin{tabular}{|c|c|c|c|}
\hline Pt ID & $\begin{array}{c}\text { HP whole tumour } \\
\text { mean(range) }\end{array}$ & $\begin{array}{c}\text { HP: epithelial } \\
\text { mean (range) }\end{array}$ & $\begin{array}{c}\text { HP: stroma } \\
\text { mean (range) }\end{array}$ \\
\hline 001 & $1(0-2)$ & $1(0-4)$ & $1(0-2)$ \\
\hline 002 & $3(2-7)$ & $8(3-13)$ & $1(0-2)$ \\
\hline 003 & $6(1-13)$ & $15(1-31)$ & $2(0-5)$ \\
\hline 004 & $10(5-14)$ & $30(12-42)$ & $4(1-5)$ \\
\hline 007 & $2(0-4)$ & $1(1-2)$ & $2(0-6)$ \\
\hline 008 & $16(11-20)$ & $20(15-26)$ & $11(6-15)$ \\
\hline 010 & $7(2-15)$ & $12(4-23)$ & $3(1-6)$ \\
\hline 012 & $15(1-30)$ & $30(12-46)$ & $7(0-19)$ \\
\hline 014 & $9(2-16)$ & $37(22-49)$ & $2(0-3)$ \\
\hline 017 & $26(20-32)$ & $39(25-53)$ & $13(9-18)$ \\
\hline
\end{tabular}
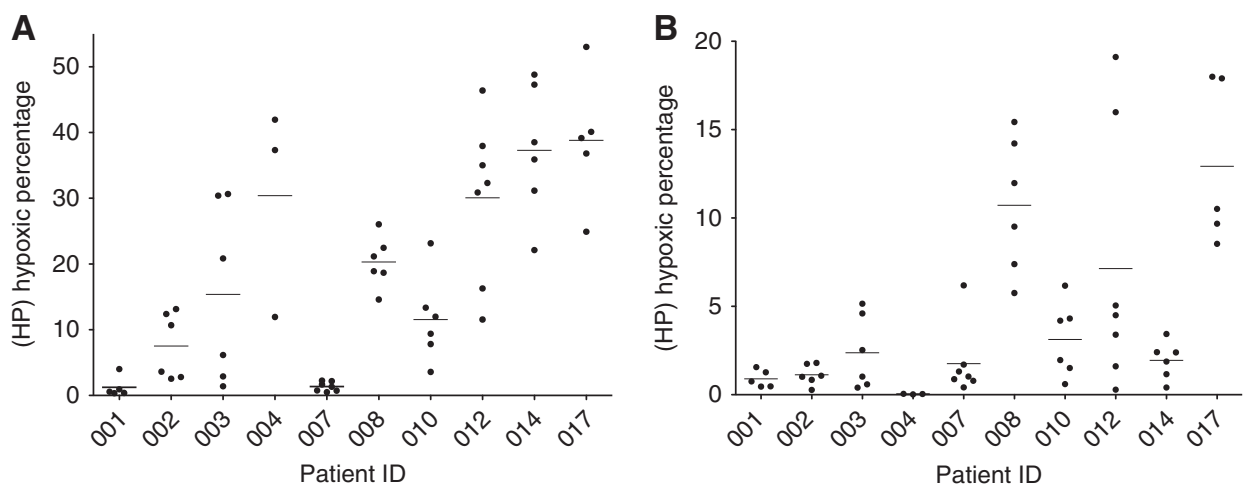

Figure 4. Hypoxia in epithelial and stromal tumour compartments. The table includes mean and range hypoxic percentages in whole tumour, and in the epithelial and stromal tumour compartments. Dot plots show the individual values and the means for the epithelial (A) and stromal (B) compartments. 
perioperative clinical trial (NCT00733746) and 1 concurrent (5-FU) chemoradiotherapy.

Quantitative estimations of the extent of stroma and hypoxia were made in the 10 resected PDACs by image analysis conducted on multiple sections per patient tumour, with each section cut from different blocks representing geographically distinct tumour regions. Number of sections analysed was 3 (in 1 patient), 5 (2 patients), 6 (5 patients) and 7 (2 patients). There was significant heterogeneity of the proportion of tumour stroma both within and across individual tumours with a range (across patients) of $38-78 \%$ (per sectional tumour area analysed); the largest intrapatient range was $41-83 \%$ (pt 008) (Figure 1).

The pattern of pimonidazole staining was cord like as previously described as characteristic of nitroimidazole markers (Raleigh et al, 1998; Raleigh et al, 1999; Evans et al, 2000). Positive staining was observed in nuclear and cytoplasmic cellular regions in both epithelial and stromal tumour compartments. Pimonidazole staining in tumour was heterogeneous across and within patient tumours, with adjacent normal pancreas demonstrating no pimonidazole staining consistent with an absence of severe tissue hypoxia (Figure 2). There was no evidence of cytotoxic treatment effect or necrosis in tumours resected from patients receiving preoperative treatment. Further, pimonidazole staining in treated tumours was similar to that observed in untreated tumours with respect to staining patterns and intensity.

The mean hypoxic percentage (defined as positive pimonidazole staining per tumoral area analysed) in whole tumours (including both epithelial and stromal tumour compartments) was $9.5 \%$ with a range of 1-26\% (Figure 3). Higher levels of hypoxia were measured in epithelial (mean 19\%; range: 1-39\%)) compared with stromal (mean $5 \%$; range: $1-13 \%$ ) regions (Figure 4 ) with a significant correlation between the two (Spearman correlation coefficient $r=0.75$; $P=0.011)$. Positive pimonidazole staining was noted in all the 4 tumours resected from patients who had received pre-operative treatment, 2 at low level ( $1 \%$ in epithelial tumour) and 2 at more moderate levels (15 and 12\%, respectively, in epithelial tumour).

Variance component analysis demonstrated greater inter- than intrapatient variability of hypoxia as indicated by positive pimonidazole staining (Table 1a). Within a given patient tumour, there was greater heterogeneity of pimonidazole staining within the stromal (in comparison with the epithelial) compartment (as indicated by the lower ICC values in tumour stroma of 0.50-0.91 vs 0.79-0.97 in epithelial tumour), whereas the greater variance between patient tumours was in the epithelial compartment (Table $1 \mathrm{~b}$ ). These data suggest that if tumour sampling is adequate to account for heterogeneity of pimonidazole staining in the epithelial component, it should be feasible to resolve biological differences related to severe hypoxia between tumours.

The average of repeat measures of a particular tumour feature provides a more accurate description of that particular characteristic than a single measurement. The greater the number of repeats, the greater the accuracy of the estimate becomes as the variance is reduced. We considered estimates of hypoxia in the epithelial tumour compartment provided by measurements made in 1-5 sections per patient tumour. As expected, there was a decrease in variance from 1 to 3 repeat measures made per tumour with further reduction as more sections were analysed. Consistent with our initial hypothesis, analysis of 4-5 sections per patient tumour appears to be able to provide some discriminatory power to differentiate among patients based on extent of pimonidazole staining (Figure 5).

\section{DISCUSSION}

Although a number of previous studies have assessed hypoxia based on the expression of surrogate markers such as HIF- $1 \alpha$ and carbonic anhydrase IX (CAIX) in pancreatectomy specimens (Couvelard et al, 2005; Sun et al, 2007; Hoffmann et al, 2008; Cheng et al, 2010), to our knowledge this is the first to use an extrinsic hypoxia tracer administered to the patient pre-operatively, and the first systematic study of sampling error related to its intratumoral heterogeneity. The accuracy of histology-based assessments of molecular markers relies on the extent of tumour sampling, which in turn should be guided by the heterogeneity of marker tissue expression (Pintilie et al, 2009). We therefore planned our analysis to include sections from multiple distinct tumour blocks. Defining 'hypoxic tissue' as that demonstrating positive pimonidazole uptake, we observed significant inter- and intratumoral heterogeneity in both epithelial and stromal tumour compartments. Variance component analysis and calculation of intraclass correlation (ICC) coefficient values helped determine the number of repeat measures required to accurately estimate hypoxia. Given that an ICC value of $>0.85$ is generally considered acceptable with respect to the robustness of measures obtained, we noted that analysis of a minimum of three sections per tumour (ICC: 0.92 ) was required to estimate hypoxia in the epithelial tumour compartment. Consistent with the higher variance of hypoxia in stroma, analysis of a minimum of 6 sections (ICC: 0.86 ) appear to be required to estimate stromal hypoxia (Table $1 \mathrm{~b}$ ).

These results raise concerns about the robustness of the assessment of tumoral hypoxia through the use of core biopsies and tissue microarrays in PDAC. They also substantiate prior work demonstrating that the accurate assessment of heterogenous (hypoxia) markers such as CAIX requires analysis of multiple core biopsies obtained systematically from geographically distant tumour locations (either from distinct tumour blocks or different

\begin{tabular}{|c|c|c|c|}
\hline & $\begin{array}{l}\text { Intrapatient } \\
\text { variance }\end{array}$ & $\begin{array}{l}\text { Interpatient } \\
\text { variance }\end{array}$ & $\begin{array}{c}\text { Ratio of } \\
\text { intra/ } \\
\text { interpatien } \\
\text { variance }\end{array}$ \\
\hline Hypoxia in whole tumour & 0.66 & 1.13 & 0.37 \\
\hline Hypoxia in epithelial tumour & 0.49 & 1.83 & 0.21 \\
\hline Hypoxia in stromal tumour & 0.74 & 0.74 & 0.5 \\
\hline
\end{tabular}

Table 1b. Intraclass coefficient (ICC) analysis estimating reliability of measures of HP made in 1-10 tumour sections per patient tumour

\begin{tabular}{|l|c|c|}
\hline Sections analysed per patient tumour & $\begin{array}{c}\text { ICC } \\
\text { epithelial }\end{array}$ & $\begin{array}{c}\text { ICC } \\
\text { stroma }\end{array}$ \\
\hline 1 & 0.79 & 0.5 \\
\hline 2 & 0.88 & 0.67 \\
\hline 3 & 0.92 & 0.75 \\
\hline 4 & 0.94 & 0.8 \\
\hline 5 & 0.95 & 0.83 \\
\hline 6 & 0.96 & 0.86 \\
\hline 7 & 0.96 & 0.88 \\
\hline 8 & 0.97 & 0.89 \\
\hline 9 & 0.97 & 0.9 \\
\hline 10 & 0.97 & 0.91 \\
\hline $\begin{array}{l}\text { Analysis demonstrates increased reliability of estimates with increasing number of sections } \\
\text { analysed, with analysis of } 2 \text { and } 6 \text { sections being required to provide an ICC greater than } \\
0.85 \text { in epithelial and stromal tumour, respectively. }\end{array}$ \\
\hline
\end{tabular}





Figure 5. Results of simulations estimating proportion of hypoxic epithelial tumour (indicated by positive pimonidazole staining in epithelial tumour cells) obtained when analysing 1-5 sections per patient tumour. In all graphs, $x$ axis indicates patient ID (in rank order of increasing levels of hypoxia) and $y$ axis indicates hypoxic fraction or proportion (log transformed) with $95 \%$ confidence interval. As anticipated, the variance in estimates of hypoxia decrease (indicated by the narrowing of the confidence intervals) as the number of sections analysed increases.

biopsies) (Thrall et al, 1997; Iakovlev et al, 2007; Maseide et al, 2008); strategies that tend to be more extensive than those currently employed (Couvelard et al, 2005; Sun et al, 2007). Therefore, there is a trade-off between minimising sampling error due to intratumoral heterogeneity of a histological marker, and the increased costs of analysing multiple tissue sections.

In contrast to a prevalent belief that PDAC are unusually hypoxic, we observed that a larger proportion of each tumour examined did not stain positively for pimonidazole. The most 'hypoxic' tumours exhibited positive staining in $20-30 \%$ of epithelial tumour areas. Furthermore, 2 out of 10 tumours showed minimal levels of pimonidazole positive staining in epithelial regions (Figure 4). Our series also included a small number of patients who had received neoadjuvant chemo/radiotherapy before surgical resection. The range of pimonidazole-detectable hypoxia was similar in the tumours from patients who had received preoperative treatment (range of epithelial hypoxia: 1-15\%) to that of the larger group. However, it is not possible in this small series to comment on the impact of treatment on the extent of tumoral hypoxia and the potential of systemic therapy and/or radiation to modulate tumoral oxygenation status is beyond the scope of the current study but worth exploring in subsequent trials.

Clinical hypoxia research has been complicated by the diverse methods and variable thresholds of oxygenation considered, as reviewed extensively (Olive et al, 2001; Bussink et al, 2003; Wilson and Hay, 2011). In the context of PDAC, the only specific study of hypoxia utilised Eppendorf electrode probes, often considered a 'gold standard' given its direct assessment of oxygenation status. Although the levels of tissue hypoxia observed in the present study are similar to those reported in nitroimidazole-based studies of other tumour types, they are much less severe than those reported using polarographic measurements made in a similar patient population (Brizel et al, 1995; Koong et al, 2000; Kaanders et al, 2002; Nordsmark et al, 2003; Chang et al, 2011). This lack of concordance has been previously observed, and attributed to differences in technique resolution, necrosis artefact and tumour heterogeneity among others (Bussink et al, 2003; Nordsmark et al, 2003; Jankovic et al, 2006; Nordsmark et al, 2006). Equally important, the bioreductive activation and binding of nitroimidazole markers like pimonidazole requires very low levels of oxygen, and therefore identifies severely hypoxic tissue at $0_{2}$ thresholds $<1-5 \mathrm{~mm} \mathrm{Hg}$. However, moderate levels of hypoxia $\left(\mathrm{pO}_{2}\right.$ of $\left.10-20 \mathrm{~mm} \mathrm{Hg}\right)$ might also be clinically relevant, given the upregulation of HIF $1 \alpha$ and related pathways at that threshold (Hockel and Vaupel, 2001a,b).

Although several hypoxia studies have been completed using nitroimidazoles in human tumours, few have attempted quantitative IHC image analysis as we have described. A small number have used quantitative immunofluorescence (IF) to study relationships amongst hypoxia, vasculature and proliferation (Kaanders et al, 2002; Hoogsteen et al, 2009; Evans et al, 2010), but IHC studies to date have focused on manual, qualitative scoring schemes (Carnell et al, 2006; Goethals et al, 2006; Hoskin et al, 2007). The potential advantages of IHC over IF include the ability to characterize hypoxia in the context of tissue morphology highly relevant for PDAC given its complex histology. Although most IHC based tissue biomarkers are evaluated manually, there is a significant literature describing inter-observer and inter-laboratory variability in the assessment of histology markers (Polley et al, 2013). Therefore, there is interest in moving towards more 
quantitative analysis of tissue-based biomarkers with a goal of reducing variability and increasing throughput (Keller et al, 2012; O'Hurley et al, 2014). However, visual evaluation by an expert pathologist continues to be the standard approach for most IHC markers, and we noted a high concordance with automated analysis of hypoxia in the epithelial tumour component (Spearman's correlation $r=0.91 ; P=0.0003$; Supplementary Figure 2).

The important conclusions from this study are that the extent of severe hypoxia in PDAC can be assessed by preoperative administration of pimonidazole, and that it is highly heterogeneous. The analysis of multiple whole sections from the same tumour shows considerable intratumoral heterogeneity, which cautions against the use of single sections or biopsy samples to assess hypoxia in PDAC. The variance of hypoxia within tumours is less than that between individual patients, supporting the feasibility to stratify patients using this approach provided that the tumour is adequately sampled.

\section{ACKNOWLEDGEMENTS}

PIMO-PANC was supported by grant funding from the Ontario Institute of Cancer Research. We thank Dr Jim Raleigh for support and useful discussions.

\section{CONFLICT OF INTEREST}

The authors declare no conflict of interest.

\section{REFERENCES}

Alexandrov LB, Nik-Zainal S, Wedge DC, Aparicio SA, Behjati S, Biankin AV, Bignell GR, Bolli N, Borg A, Borresen-Dale AL, Boyault S, Burkhardt B, Butler AP, Caldas C, Davies HR, Desmedt C, Eils R, Eyfjord JE, Foekens JA, Greaves M, Hosoda F, Hutter B, Ilicic T, Imbeaud S, Imielinski M, Jager N, Jones DT, Jones D, Knappskog S, Kool M, Lakhani SR, Lopez-Otin C, Martin S, Munshi NC, Nakamura H, Northcott PA, Pajic M, Papaemmanuil E, Paradiso A, Pearson JV, Puente XS, Raine K, Ramakrishna M, Richardson AL, Richter J, Rosenstiel P, Schlesner M, Schumacher TN, Span PN, Teague JW, Totoki Y, Tutt AN, Valdes-Mas R, van Buuren MM, van 't Veer L, Vincent-Salomon A, Waddell N, Yates LR, Zucman-Rossi J, Futreal PA, McDermott U, Lichter P, Meyerson M, Grimmond SM, Siebert R, Campo E, Shibata T, Pfister SM, Campbell PJ, Stratton MR (2013) Signatures of mutational processes in human cancer. Nature 500(7463): $415-421$.

Armstrong T, Packham G, Murphy LB, Bateman AC, Conti JA, Fine DR, Johnson CD, Benyon RC, Iredale JP (2004) Type I collagen promotes the malignant phenotype of pancreatic ductal adenocarcinoma. Clin Cancer Res 10(21): 7427-7437.

Brizel DM, Rosner GL, Prosnitz LR, Dewhirst MW (1995) Patterns and variability of tumor oxygenation in human soft tissue sarcomas, cervical carcinomas, and lymph node metastases. Int J Radiat Oncol Biol Phys 32(4): 1121-1125.

Brown JM, Wilson WR (2004) Exploiting tumour hypoxia in cancer treatment. Nat Rev Cancer 4(6): 437-447.

Buchler P, Reber HA, Lavey RS, Tomlinson J, Buchler MW, Friess H, Hines OJ (2004) Tumor hypoxia correlates with metastatic tumor growth of pancreatic cancer in an orthotopic murine model. J Surg Res 120(2): 295-303.

Bussink J, Kaanders J, van der Kogel A (2003) Tumor hypoxia at the microregional level: clinical relevance and predictive value of exogenous and endogenous hypoxic cell markers. Radiother Oncol 67(1): 3-15.

Carnell DM, Smith RE, Daley FM, Saunders MI, Bentzen SM, Hoskin PJ (2006) An immunohistochemical assessment of hypoxia in prostate carcinoma using pimonidazole: implications for radioresistance. Int J Radiat Oncol Biol Phys 65(1): 91-99.

Chang Q, Jurisica I, Do T, Hedley DW (2011) Hypoxia predicts aggressive growth and spontaneous metastasis formation from orthotopically grown primary xenografts of human pancreatic cancer. Cancer Res 71(8): 3110-3120.

Cheng K, Ho K, Stokes R, Scott C, Lau SM, Hawthorne WJ, O'Connell PJ, Loudovaris T, Kay TW, Kulkarni RN, Okada T, Wang XL, Yim SH, Shah Y, Grey ST, Biankin AV, Kench JG, Laybutt DR, Gonzalez FJ, Kahn CR, Gunton JE (2010) Hypoxia-inducible factor-1alpha regulates beta cell function in mouse and human islets. J Clin Invest 120(6): 2171-2183.

Couvelard A, O'Toole D, Leek R, Turley H, Sauvanet A, Degott C, Ruszniewski P, Belghiti J, Harris AL, Gatter K, Pezzella F (2005) Expression of hypoxia-inducible factors is correlated with the presence of a fibrotic focus and angiogenesis in pancreatic ductal adenocarcinomas. Histopathology 46(6): 668-676.

Evans SM, Hahn S, Pook DR, Jenkins WT, Chalian AA, Zhang P, Stevens C, Weber R, Weinstein G, Benjamin I, Mirza N, Morgan M, Rubin S, McKenna WG, Lord EM, Koch CJ (2000) Detection of hypoxia in human squamous cell carcinoma by EF5 binding. Cancer Res 60(7): 2018-2024.

Evans SM, Jenkins KW, Chen HI, Jenkins WT, Judy KD, Hwang WT, Lustig RA, Judkins AR, Grady MS, Hahn SM, Koch CJ (2010) The relationship among hypoxia, proliferation, and outcome in patients with de novo glioblastoma: a pilot study. Transl Oncol 3(3): 160-169.

Fusaroli P, Spada A, Mancino MG, Caletti G (2010) Contrast harmonic echo-endoscopic ultrasound improves accuracy in diagnosis of solid pancreatic masses. Clin Gastroenterol Hepatol 8(7): 629-34 e1-2.

Fyles A, Milosevic M, Hedley D, Pintilie M, Levin W, Manchul L, Hill RP (2002) Tumor hypoxia has independent predictor impact only in patients with node-negative cervix cancer. J Clin Oncol 20(3): 680-687.

Goethals L, Debucquoy A, Perneel C, Geboes K, Ectors N, De Schutter H, Penninckx F, McBride WH, Begg AC, Haustermans KM (2006) Hypoxia in human colorectal adenocarcinoma: comparison between extrinsic and potential intrinsic hypoxia markers. Int J Radiat Oncol Biol Phys 65(1): 246-254.

He Y, Liu XD, Chen ZY, Zhu J, Xiong Y, Li K, Dong JH, Li X (2007) Interaction between cancer cells and stromal fibroblasts is required for activation of the uPAR-uPA-MMP-2 cascade in pancreatic cancer metastasis. Clin Cancer Res 13(11): 3115-3124.

Hockel M, Vaupel P (2001a) Biological consequences of tumor hypoxia. Semin Oncol 28(2 Suppl 8): 36-41.

Hockel M, Vaupel P (2001b) Tumor hypoxia: definitions and current clinical, biologic, and molecular aspects. J Natl Cancer Inst 93(4): 266-276.

Hoffmann AC, Mori R, Vallbohmer D, Brabender J, Klein E, Drebber U, Baldus SE, Cooc J, Azuma M, Metzger R, Hoelscher AH, Danenberg KD, Prenzel KL, Danenberg PV (2008) High expression of HIFla is a predictor of clinical outcome in patients with pancreatic ductal adenocarcinomas and correlated to PDGFA, VEGF, and bFGF. Neoplasia 10(7): 674-679.

Hoogsteen IJ, Lok J, Marres HA, Takes RP, Rijken PF, van der Kogel AJ, Kaanders JH (2009) Hypoxia in larynx carcinomas assessed by pimonidazole binding and the value of CA-IX and vascularity as surrogate markers of hypoxia. Eur J Cancer 45(16): 2906-2914.

Hoskin PJ, Carnell DM, Taylor NJ, Smith RE, Stirling JJ, Daley FM, Saunders MI, Bentzen SM, Collins DJ, d'Arcy JA, Padhani AP (2007) Hypoxia in prostate cancer: correlation of BOLD-MRI with pimonidazole immunohistochemistry-initial observations. Int J Radiat Oncol Biol Phys 68(4): 1065-1071.

Iakovlev VV, Pintilie M, Morrison A, Fyles AW, Hill RP, Hedley DW (2007) Effect of distributional heterogeneity on the analysis of tumor hypoxia based on carbonic anhydrase IX. Lab Invest 87(12): 1206-1217.

Jankovic B, Aquino-Parsons C, Raleigh JA, Stanbridge EJ, Durand RE, Banath JP, MacPhail SH, Olive PL (2006) Comparison between pimonidazole binding, oxygen electrode measurements, and expression of endogenous hypoxia markers in cancer of the uterine cervix. Cytometry $B$ Clin Cytom 70(2): 45-55.

Jones S, Zhang X, Parsons DW, Lin JC, Leary RJ, Angenendt P, Mankoo P, Carter H, Kamiyama H, Jimeno A, Hong SM, Fu B, Lin MT, Calhoun ES, Kamiyama M, Walter K, Nikolskaya T, Nikolsky Y, Hartigan J, Smith DR, Hidalgo M, Leach SD, Klein AP, Jaffee EM, Goggins M, Maitra A, Iacobuzio-Donahue C, Eshleman JR, Kern SE, Hruban RH, Karchin R, Papadopoulos N, Parmigiani G, Vogelstein B, Velculescu VE, Kinzler KW (2008) Core signaling pathways in human pancreatic cancers revealed by global genomic analyses. Science 321(5897): 1801-1806.

Kaanders JH, Wijffels KI, Marres HA, Ljungkvist AS, Pop LA, van den Hoogen FJ, de Wilde PC, Bussink J, Raleigh JA, van der Kogel AJ (2002) Pimonidazole binding and tumor vascularity predict for treatment outcome in head and neck cancer. Cancer Res 62(23): 7066-7074. 
Keller B, Chen W, Gavrielides MA (2012) Quantitative assessment and classification of tissue-based biomarker expression with color content analysis. Arch Pathol Lab Med 136(5): 539-550.

Koong AC, Mehta VK, Le QT, Fisher GA, Terris DJ, Brown JM, Bastidas AJ, Vierra M (2000) Pancreatic tumors show high levels of hypoxia. Int J Radiat Oncol Biol Phys 48(4): 919-922.

Maseide K, Pintilie M, Kandel R, Hill RP (2008) Can sparsely and heterogeneously expressed proteins be detected using tissue microarrays? A simulation study of the hypoxia marker carbonic anhydrase IX (CA IX) in human soft tissue sarcoma. Pathol Res Pract 204(3): 175-183.

Matsubara H, Itoh A, Kawashima H, Kasugai T, Ohno E, Ishikawa T, Itoh Y, Nakamura Y, Hiramatsu T, Nakamura M, Miyahara R, Ohmiya N, Ishigami M, Katano Y, Goto H, Hirooka Y (2011) Dynamic quantitative evaluation of contrast-enhanced endoscopic ultrasonography in the diagnosis of pancreatic diseases. Pancreas 40(7): 1073-1079.

Neesse A, Michl P, Frese KK, Feig C, Cook N, Jacobetz MA, Lolkema MP, Buchholz M, Olive KP, Gress TM, Tuveson DA (2011) Stromal biology and therapy in pancreatic cancer. Gut 60(6): 861-868.

Nordsmark M, Loncaster J, Aquino-Parsons C, Chou SC, Gebski V, West C, Lindegaard JC, Havsteen H, Davidson SE, Hunter R, Raleigh JA, Overgaard J (2006) The prognostic value of pimonidazole and tumour pO2 in human cervix carcinomas after radiation therapy: a prospective international multi-center study. Radiother Oncol 80(2): 123-131.

Nordsmark M, Loncaster J, Aquino-Parsons C, Chou SC, Ladekarl M, Havsteen H, Lindegaard JC, Davidson SE, Varia M, West C, Hunter R, Overgaard J, Raleigh JA (2003) Measurements of hypoxia using pimonidazole and polarographic oxygen-sensitive electrodes in human cervix carcinomas. Radiother Oncol 67(1): 35-44.

O’Hurley G, Sjostedt E, Rahman A, Li B, Kampf C, Ponten F, Gallagher WM, Lindskog C (2014) Garbage in, garbage out: a critical evaluation of strategies used for validation of immunohistochemical biomarkers. Mol Oncol 8(4): 783-798.

Olive KP, Jacobetz MA, Davidson CJ, Gopinathan A, McIntyre D, Honess D, Madhu B, Goldgraben MA, Caldwell ME, Allard D, Frese KK, Denicola G, Feig C, Combs C, Winter SP, Ireland-Zecchini H, Reichelt S, Howat WJ, Chang A, Dhara M, Wang L, Ruckert F, Grutzmann R, Pilarsky C, Izeradjene K, Hingorani SR, Huang P, Davies SE, Plunkett W, Egorin M, Hruban RH, Whitebread N, McGovern K, Adams J, Iacobuzio-Donahue C, Griffiths J, Tuveson DA (2009) Inhibition of Hedgehog signaling enhances delivery of chemotherapy in a mouse model of pancreatic cancer. Science 324(5933): 1457-1461.

Olive PL, Banath JP, Aquino-Parsons C (2001) Measuring hypoxia in solid tumours-is there a gold standard? Acta Oncol 40(8): 917-923.

Overgaard J (2011) Hypoxic modification of radiotherapy in squamous cell carcinoma of the head and neck-a systematic review and meta-analysis. Radiother Oncol 100(1): 22-32.

Pintilie M, Iakovlev V, Fyles A, Hedley D, Milosevic M, Hill RP (2009) Heterogeneity and power in clinical biomarker studies. J Clin Oncol 27(9): $1517-1521$.

Polley MY, Leung SC, McShane LM, Gao D, Hugh JC, Mastropasqua MG, Viale G, Zabaglo LA, Penault-Llorca F, Bartlett JM, Gown AM, Symmans WF, Piper T, Mehl E, Enos RA, Hayes DF, Dowsett M,
Nielsen TO (2013) An international Ki67 reproducibility study. J Natl Cancer Inst 105(24): 1897-1906.

Provenzano PP, Cuevas C, Chang AE, Goel VK, Von Hoff DD, Hingorani SR (2012) Enzymatic targeting of the stroma ablates physical barriers to treatment of pancreatic ductal adenocarcinoma. Cancer Cell 21(3): 418-429.

Rahib L, Smith BD, Aizenberg R, Rosenzweig AB, Fleshman JM, Matrisian LM (2014) Projecting cancer incidence and deaths to 2030: the unexpected burden of thyroid, liver, and pancreas cancers in the United States. Cancer Res 74(11): 2913-2921.

Raleigh J, Calkins-Adams D, Rinker L, Ballenger C, Weissler M, Fowler Jr WC, Novotny DB, Varia MA (1998) Hypoxia and vascular endothelial growth factor expression in human squamous cell carcinomas using pimonidazole as a hypoxia marker. Cancer Res 58(17): 3765-3768.

Raleigh JA, Chou SC, Arteel GE, Horsman MR (1999) Comparisons among pimonidazole binding, oxygen electrode measurements, and radiation response in $\mathrm{C} 3 \mathrm{H}$ mouse tumors. Radiat Res 151(5): 580-589.

Raleigh JA, Miller GG, Franko J, Chapman JD (1992) Immunochemical detection of hypoxia in normal and tumor tissue. US patent no. 5086068. 4 February 1992.

Rischin D, Hicks R, Fisher R, Binns D, Corry J, Porceddu S, Peters L (2006) Prognostic significance of [18F]-misonidazole positron emission tomography-detected tumor hypoxia in patients with advanced head and neck cancer randomly assigned to chemoradiation with or without tirapazamine: a substudy of Trans-Tasman Radiation Oncology Group Study 98.02. J Clin Oncol 24(13): 2098-2104.

Spivak-Kroizman TR, Hostetter G, Posner R, Aziz M, Hu C, Demeure MJ, Von Hoff D, Hingorani SR, Palculict TB, Izzo J, Kiriakova GM, Abdelmelek M, Bartholomeusz G, James BP, Powis G (2013) Hypoxia triggers hedgehog-mediated tumor-stromal interactions in pancreatic cancer. Cancer Res 73(11): 3235-3247.

Sun HC, Qiu ZJ, Liu J, Sun J, Jiang T, Huang KJ, Yao M, Huang C (2007) Expression of hypoxia-inducible factor-1 alpha and associated proteins in pancreatic ductal adenocarcinoma and their impact on prognosis. Int J Oncol 30(6): 1359-1367.

Thrall DE, Rosner GL, Azuma C, McEntee MC, Raleigh JA (1997) Hypoxia marker labeling in tumor biopsies: quantification of labeling variation and criteria for biopsy sectioning. Radiother Oncol 44(2): 171-176.

Varia MA, Calkins-Adams DP, Rinker LH, Kennedy AS, Novotny DB, Fowler Jr WC, Raleigh JA (1998) Pimonidazole: a novel hypoxia marker for complementary study of tumor hypoxia and cell proliferation in cervical carcinoma. Gynecol Oncol 71(2): 270-277.

Vaupel P (2008) Hypoxia and aggressive tumor phenotype: implications for therapy and prognosis. Oncologist 13(Suppl 3): 21-26.

Wilson WR, Hay MP (2011) Targeting hypoxia in cancer therapy. Nat Rev Cancer 11(6): 393-410.

This work is published under the standard license to publish agreement. After 12 months the work will become freely available and the license terms will switch to a Creative Commons AttributionNonCommercial-Share Alike 4.0 Unported License.

Supplementary Information accompanies this paper on British Journal of Cancer website (http://www.nature.com/bjc) 